\title{
TRACKS MATCHING BASED ON AN ACOUSTIC SPECTRUM SIGNATURE DETECTION AND A MULTI-FRAME FUSION ALGORITHMS
}

\author{
Dahai Cheng ${ }^{1}$, Huigang $\mathrm{Xu}^{1}$ and Ruiliang Gong ${ }^{2}$, Huan Huang ${ }^{2}$ \\ ${ }^{1}$ School of Electric and Automatic Engineering, Changshu Institute of \\ Technology, Changshu, Jiangsu, China. 215500 \\ ${ }^{2}$ Changshu Ruite Electric Co. Ltd 2nd Changshu, Jiangsu, China. 215500
}

\begin{abstract}
In this paper, an acoustic spectrum signature tracks matching algorithm based on the Manhattan distance and the Euclidean distance of signature vectors, and a multi-frame fusion algorithm are proposed for reliable real time detection and matching of boat generated acoustic signal spectrum signatures. The experiments results have shown that the proposed tracks matching algorithm has the ability to discriminate the tracks from different ships and the ability of matching of the tracks from the same ship; and the spectrum signature detection algorithm has captured the critical features of ship generated acoustic signals. In the process of signal spectrum signature detection, the observation of time and frequency space is structured by dividing input digitalized acoustic signal into multiple frames and each frame is transformed into the frequency domain by FFT. Then, a normalization of signal spectrum vector is carried out to make the detection process more robust. After that, an adaptive median Constant False Alarm Rate (AMCFAR) algorithm is used for the detection and extraction of boat generated spectrum signature, in which an extreme low constant false alarm rate is kept with relative high detection rate. Finally, the frame detections are accumulated to build up the track spectrum signatures.
\end{abstract}

\section{KEYWORDS}

Tracks Matching, Multi-Frame Fusion, Time-Frequency Observation Space, Spectrum Signature Detection

\section{INTRODUCTION}

Tracks matching plays an important role at detection and tracking of marine vehicles, including some underwater targets, such as submarine etc. The discrimination of targets is based on the comparison of the given spectra with the reference spectra available as endmembers in a spectral library. The comparison is done using the similarity as a criterion [1-4]. Stochastic measures such as spectral information divergence consider the spectral band to band variability as a result of uncertainty incurred by randomness. The spectrum can be modelled as a probability distribution so that the spectral properties can be further described by statistical moments of any order [1].

David C. Wyld et al. (Eds) : CSITY, DTMN, NWCOM, SIGPRO - 2018 pp. 73-92, 2018. @ CS \& IT-CSCP 2018

DOI : $10.5121 /$ csit.2018.81107 
The hybrid approaches of spectral angle mapper and spectral information divergence is found to increase the discriminatory power as against the individual measures [4].

Detection and extraction of underwater acoustic signal play an extremely important role in marine vehicle tracking and is one of the key technologies in underwater source detection, location, tracking, recognition and as well as acoustic communication [5-11]. Energy detection, feature detection and matched filter detection are commonly used range from high detection performance to low computation complexity, which can usually work well to some extent. Among these researches of the underwater passive detection methods, energy detection is mostly discussed and used in practical for its merits of lowest computational cost and easy to realize, while shows a poor performance under low SNR marine environment. However, over the past several decades, the marine environment has been more complexed in both natural and anthropogenic influences that ambient noise revealed. And as a consequence, the existing underwater passive detection methods are facing serious challenges, for which advanced detection scheme with better performance is still worthy investigating, especially under low signal-to-noise ratio (SNR) region [12-14].

This paper is focused on the research of theoretic algorithm development and experiments of tracks matching, and automatic detection ship spectrum signature based on boat generated acoustic signals from hydrophone ([15-18]). In this paper, an observation space is created by dividing input acoustic signals into multiple frames, with each frame sampled and transformed into the frequency domain. Then, an Adaptive Median Constant False Alarm Rate algorithm [19] is used for automatic target detection of boat-generated acoustic signals in each frame to provide a low constant false alarm rate with relatively high detection rate. Finally, the track signal spectrum signature is built up by a multi-frame detection vector fusion algorithm, in which the signal-to-noise ratio (SNR) in the observation period has been increased in the detection phase. The proposed algorithms have been tested on real boat generated acoustic signals obtained from a hydrophone.

\section{Multi-Frame Spectrum Signature Detection in Time FREQUENCY DOMAIN}

In this section, the observation is created first. Then, an adaptive signal spectrum components detection algorithm is used for the signature detection.

\subsection{Multi-Frame Spectrum Observation Space}

The observation space for multi-frame spectrum detection and fusion is created by dividing input time domain signal into multiple frames, and each frame in transformed into frequency domain by using FFT, which is shown in Fig. 1, in which $\mathrm{x}$-direction represent number of frequency bins, and the y-direction represent time or number of frames.

\subsection{Adaptive Spectrum Signature Detection Algorithm}

An adaptive CFAR spectrum signature detection algorithm used in this paper are structured based on the idea of median CFAR thresholding, and the normalization operation based NeymanPearson (NP) criterion ([19-22]), which is widely adopted for signal detection application in either radar or sonar systems. 


\section{Frequency (Number of Frequency Bins)}

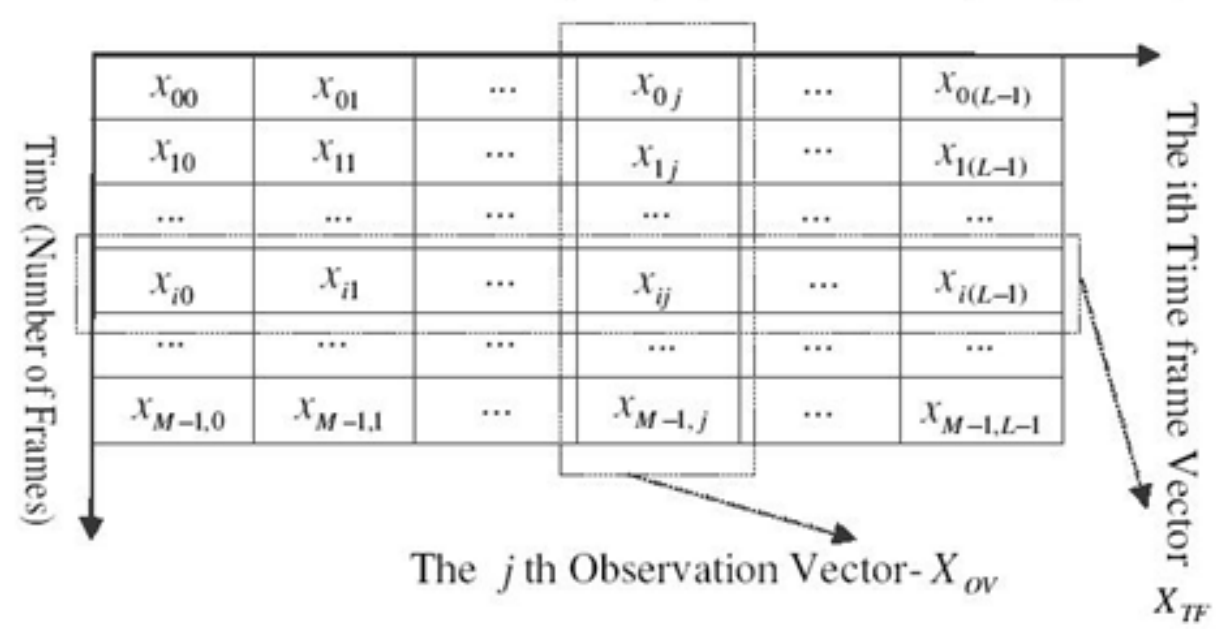

Figure 1. Observation space for the acoustic signal spectrum

The whole multi-frame acoustic signal processing and detection algorithms employed in the paper is shown in Fig. 2. In which, the acoustic signals from hydrophone are sampled and converted into digital signals based on the Nyquist Sampling Theorem (Criterion) ([24-27]). In our experiments, the signals are originally sampled at $44.1 \mathrm{kHz}$, then resampled to $2048 \mathrm{~Hz}$, so the maximum frequency range in our scope is $1024 \mathrm{~Hz}$. After that, the digital signal is divided into multiple frames with $\mathrm{T}=0.5$ seconds for each frame, and the data processing period in digital format is $\mathrm{N}=1024$.

The digital signals are then transformed into the frequency domain by using FFT (Fast Fourier Transform). Since the data processing period is 1024, we chose the same length, i.e. 1024 points as FFT length.

Normally DC is the strongest component in the boat generated acoustic signals, which does not carry any useful information, but will affect the later processing, so it is necessary to remove DC component first.

We assume that the boat generated acoustic signals are stable random process during the observation period, such as 15 to 20 seconds.

In order to deal with various input signal strength and make the automatic target spectrum signature detection more robust, the normalization is performed in the frequency domain for the multi-frame spectrum fused vector by its magnitude. At this stage, each element in the multiframe fusion vector is divided by the magnitude of the vector (geometric length). After normalization, a magnitude scaling factor of $40 \mathrm{~dB}$ (100 times) is used to give the signal a more practical range. 


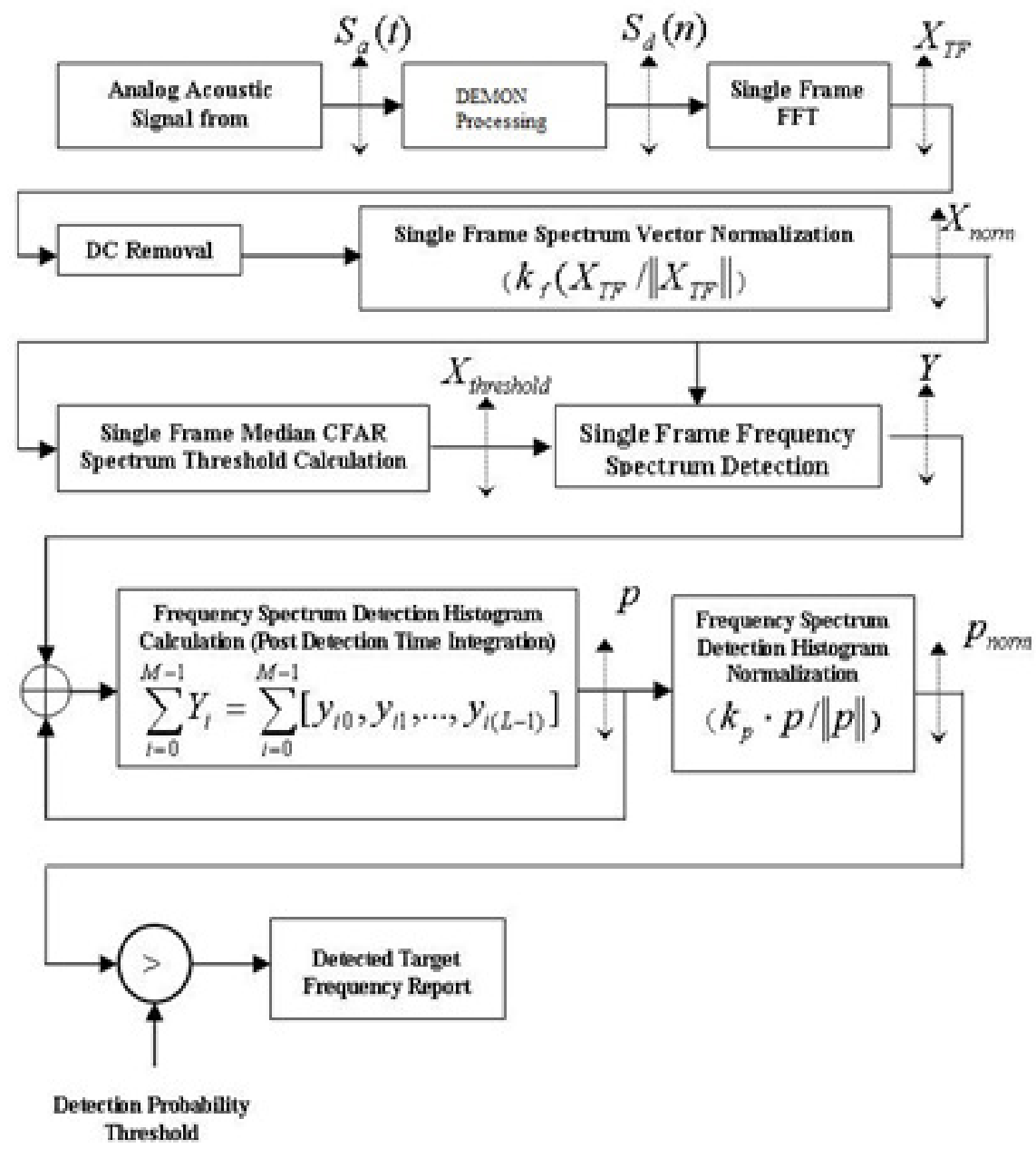

Figure 2. Adaptive Spectrum Signature Detection Multi-Frame Fusion Algorithms

The multi-frame spectrum fusion vector normalization is described as follows

$$
X_{T F}^{\text {norm }}=k_{f} \cdot X_{T F} /\left\|X_{T F}\right\|=k_{f} \cdot \frac{\left[x_{0}, x_{1}, \cdots, x_{(L-1)}\right]^{T}}{\sqrt{x_{0}^{2}+x_{1}^{2}+\cdots+x_{(L-1)}^{2}}}
$$

in which $\mathrm{L}$ is the number of frequency bins in the fusion vector and $\mathrm{kf}$ is the scaling factor.

In traditional sonar systems, signal detection is realized by a constant thresholding. Instead, in our approach, a floating threshold vector for the multi-frame fusion vector is calculated based on an Adaptive Median CFAR algorithm, in which the threshold for each frequency bin is adapted by the median value over a sliding window. This is performed in two steps: in the first step, the median threshold of the normalized spectrum vector is subtracted from the original normalized spectrum vector. In the second step, the difference calculated in the first step is compared with a constant CFAR threshold $(\Delta)$, and if the difference is big enough to cross the constant threshold, the bin is reported as the target frequency, otherwise, it is reported as noise component. The 
parameter is called sensitivity, as the bigger the $\Delta$ is, the less sensitive our detection system is on weak signals. Since the CFAR threshold in each bin is adapted to its neighbourhood background noise, it will keep our automatic spectrum signature detection system at a very low and constant false alarm rate. In the following, the AMCFAR algorithm is described in details.

The Median Constant False Alarm Rate (Median CFAR) threshold vector,

$$
\begin{aligned}
& X_{\text {threshold }}=\left[x_{\text {threshold }, 0}, x_{\text {threshold }, 1}, \cdots, x_{\text {threshold }, L-1}\right]^{T} \\
& =\left[x_{\text {threshold }, i}\right]^{T}
\end{aligned}
$$

is calculated by feeding into a Median filter (whose properties and size will be discussed in the next section) as follows:

$$
x_{\text {threshold }, i}=\operatorname{Median}\left\{x_{\text {norm }, i-k}, \cdots, x_{\text {norm }, i}, \cdots, x_{\text {norm }, i+k}\right\}
$$

The Median filter size is $(2 k+1)$, with $k=1,2,3$. In order to deal with the boundary case, both the input signal spectrum vector and the threshold vector are treated as wrapped period signals. The multi-frame fusion vector detection is based on the comparison of the difference between vectors $Y_{\text {norm }}$ and $Y_{\text {norm }}^{\text {threshold }}$ against a threshold, with the output being a binary vector:

$$
Y_{i}=\left[y_{i 0}, y_{i 1}, \cdots, y_{i(L-1)}\right]^{T}
$$

with $Y_{j}$, where $\mathrm{j}=0,1, \ldots, \mathrm{L}-1$ given by:

$$
y_{i j}=\left\{\begin{array}{l}
1,\left(\begin{array}{l}
x_{\text {norm }}, i j \\
0, x_{\text {threshold }}, i j
\end{array}\right) \geq \Delta \\
0 \text { otherwise }
\end{array}\right.
$$

The $y_{i j}$ values are either equal to 1 or 0 , which are the detected frequencies based on the multiframe fusion detection. As boat-generated signals typically last at least 20 to 30 minutes, it is reasonable to treat the signal stable random process in the observation period of 15 to 20 seconds, and it has been proven that the proposed algorithm can significantly improve the detection rate.

In order to increase the signal-to-noise ratio in frequency domain, a multi-frame detection vector fusion is used in the paper as shown in Equation 6.

$$
\begin{aligned}
& p=\left[p_{0,} p_{1, \ldots,} p_{(L-1)}\right] \\
& =\sum_{i=0}^{M-1} Y_{i}=\sum_{i=0}^{M-1}\left[y_{i 0}, y_{i 1}, \ldots, y_{i(L-1)}\right]
\end{aligned}
$$

Where $\mathrm{M}$ is the number of frames in the observation period, and $\mathrm{L}$ is the number of frequency bins in each frame. 


\section{SIGNATURE TRACKS MATCHING}

The discrimination of targets is based on the comparison of the detected signature spectrum with the reference spectra stored in our spectral database, and the comparison is carried out by using the similarity or distance between spectrum signature vectors. The tracks matching algorithm is shown in Fig. 3, in which we can see that the final decision of whether the two signature tracks are from different ships or from the same ship is based on blocks matching and final fusion, and each block consisted of 15 frames, and each frame is a 1024 point spectrum vector.

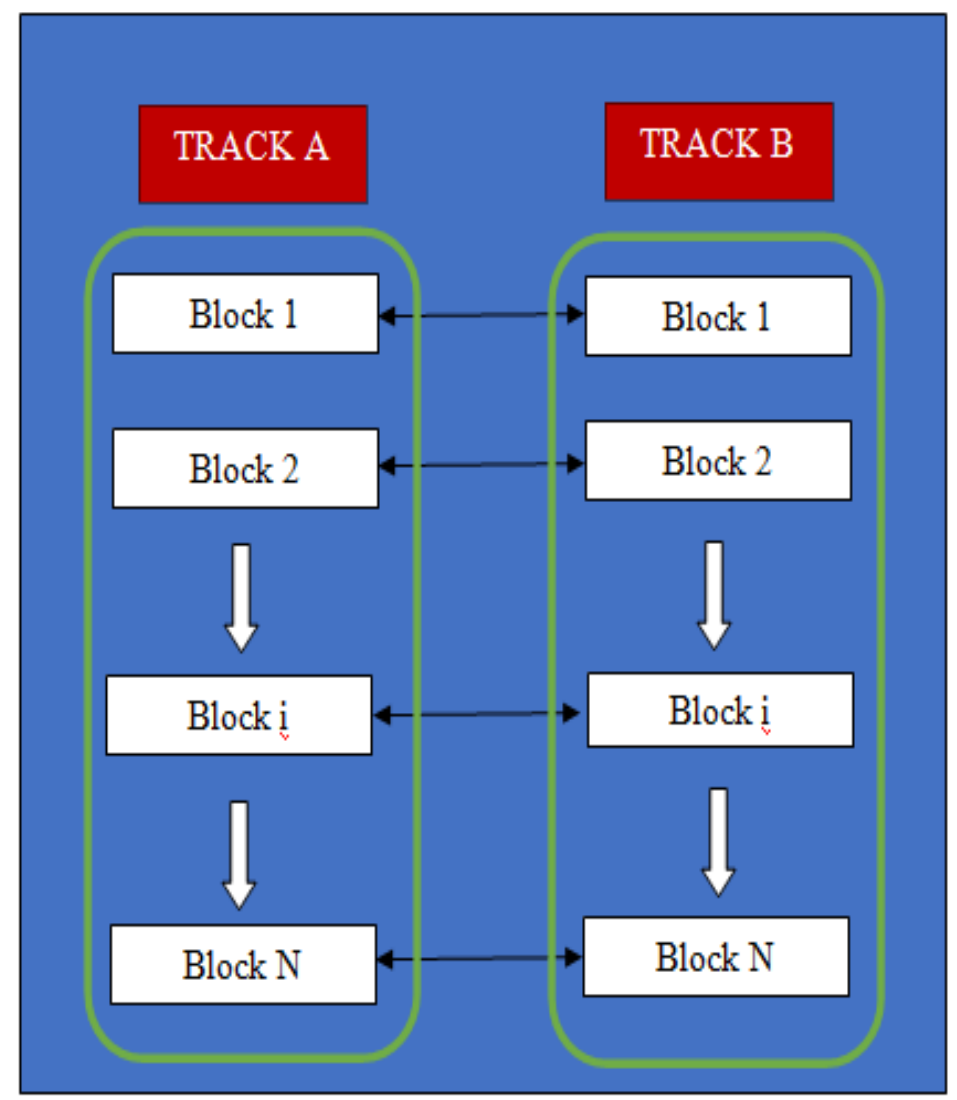

Figure 3. Tracks matching algorithm

The blocks matching is based on the similarity or distance measurements between two spectrum signature vectors, which include Manhattan and Euclidean distance distances in our proposed tracking algorithms.

Manhattan distance: Manhattan distance is also called taxicab distance. A taxicab geometry is a form of geometry in which the usual distance function or metric of Euclidean geometry is replaced by a new metric in which the distance between two points is the sum of the absolute differences of their Cartesian coordinates. The taxicab metric is also known as rectilinear distance, $L 1$ distance, $L 1$ distance or $\ell^{1}$ norm (see $L^{p}$ space), snake distance, city block distance, Manhattan distance or Manhattan length, with corresponding variations in the name of the geometry. The latter names allude to the grid layout of most streets on the island of Manhattan, 
which causes the shortest path a car could take between two intersections in the borough to have length equal to the intersections' distance in taxicab geometry.

Formal definition: The taxicab distance, $\mathrm{d}\left(\mathrm{Sig}_{1}, \mathrm{Sig}_{2}\right)$, between two vectors $\mathrm{Sig}_{1}$ and $\mathrm{Sig}_{2}$ in an N dimensional real vector space with fixed Cartesian coordinate system, is the sum of the lengths of the projections of the line segment between the points onto the coordinate axes. More formally,

$$
d\left(S g_{1}, \mathcal{S g}\right)=\left|S g_{1,1}-\mathcal{S} g_{2,1}\right|+\left|S g_{1,2}-\mathcal{S} g_{2,2}\right|+\cdots+\left|S g_{1, N}-\mathcal{S g} g_{2, N}\right|=\sum_{i=1}^{N}\left|S g_{1, i}-\mathcal{S} g_{2, i}\right|
$$

where $\operatorname{Sig}_{1}$ and $\operatorname{Sig}_{2}$ are vectors $\operatorname{Sig}_{1}=\left(\operatorname{Sig}_{11}, \operatorname{Sig}_{12}, \ldots, \operatorname{Sig}_{I N}\right)$, and $\operatorname{Sig}_{2}=\left(\operatorname{Sig}_{21}, \operatorname{Sig}_{22}, \ldots, \operatorname{Sig}_{2 N}\right)$.

For example, in the plane, the taxicab distance between $S i g_{1}$ and $S i g_{2}$ is $\sum_{i=1}^{N}\left|S i g_{1, i}-S i g_{2, i}\right|$.

Euclidean distance: In similarity measurement, we also use the Euclidean distance or Euclidean metric, which is the "ordinary" straight-line distance between two points in Euclidean space. With this distance, Euclidean space becomes a metric space. The associated norm is called the Euclidean norm, or a generalized term for the Euclidean norm is the $\mathrm{L}^{2}$ norm or $\mathrm{L}^{2}$ distance.

The Euclidean distance between signature points Sig1 and Sig2 is the length of the line segment connecting them .In Cartesian coordinates, if the two spectrum signatures $\operatorname{Sig}_{1}=\left(\operatorname{Sig}_{11}, \operatorname{Sig}_{12}, \ldots, \operatorname{Sig}_{I N}\right)$ and $\operatorname{Sig}_{2}=\left(\operatorname{Sig}_{21}, \operatorname{Sig}_{22}, \ldots, \operatorname{Sig}_{2 N}\right)$ are two points in Euclidean $N-$ space, then the distance (d) from $\mathrm{Sig}_{1}$ to $\mathrm{Sig}_{2}$, or from $\mathrm{Sig}_{1}$ to $\mathrm{Sig}_{2}$ is given by the Pythagorean formula

$$
\left.d S g_{1}, S g_{2}\right)=\sqrt{\left(S g_{1,1}-S g_{2,1}\right)^{2}+\left(S g_{1,2}-S g_{2,2}\right)^{2}+\cdots+\left(S g_{1, N}-S g_{2, N}\right)^{2}}=\sqrt{\sum_{i=1}^{N}\left(S g_{1, i}-S g_{2, i}\right)^{2}}
$$

Stochastic measures such as spectral information divergence consider the spectral band to band variability as a result of uncertainty incurred by randomness. The spectrum can be modelled as a probability distribution so that the spectral properties can be further described by statistical moments of any order. The hybrid approaches of spectral angle mapper and spectral information divergence is found to increase the discriminatory power as against the individual measures.

\section{RELATED WORKS AND DISCUSSION}

In this paper, a tracks matching algorithm based on Manhattan distance and Euclidean distance, a multi-frame detection fusion and an adaptive signal spectrum detection algorithms are proposed, in which the tracks from different ships can be easily discriminated by both Manhattan distance and Euclidean distance; the spectrum signature detection algorithm captured the critical features, with a low false alarm and relative high detection rates in the frequency domain; and the signalto-noise ratio in each frequency bin is increased by a multi-frame fusion algorithm. The input acoustic signal is sampled and divided into multiple frames. Then each frame is transformed into the frequency domain by using FFT. After that, the target generated frequency spectrum will be detected based on accumulated and normalized spectrum vector by the proposed adaptive algorithm, in which the basic idea of the detection algorithm is that of using, for each frequency bin, different, adaptive CFAR (Constant False Alarm Rate) thresholds [8] rather than a single, 
constant threshold (which is often the case in acoustic systems), which is described below. Then, a multi-frame fusion on is carried out by accumulating single frame detection result vectors in the observation period, for example, in 15 seconds, equivalent of 30 frames in our experiments. Finally, the tracks matching is carried out based on the given Manhattan distance and Euclidean distance measurements.

The basic idea of CFAR thresholding is that every single threshold of each frequency bin is computed based on the surrounding background noise. The higher the background noise, the higher the threshold is set. Moreover, our algorithm uses a median filter window centered at each frequency bin to adapt the threshold value. To the best of our knowledge, while this idea is often used in radar system to obtain lower false alarm rate with relatively higher target detection rate, it is applied here for the first time to sonar-generated acoustic signals.

Since the Median Filter is good at removing high frequency spot noise, it is a very effective way to calculate the threshold vector, which is independent of specific signals. As such, the Adaptive Median CFAR algorithm proves superior to other common approaches such as constant thresholds or average-based thresholds. The major advantage of the Median filter is in its ability

to remove interferences such as strong signal or noise spikes without affecting the sharpness of edges (retaining sharp edges after filtering). Conversely, with an Averaging Low Pass Filter, which is equivalent to the Average CFAR algorithm, sharp edges will be blurred after filtering. Moreover, every bin in the averaging window will affect the threshold value, especially when the signal or a noise spike is strong. Evidence of the superiority of the median filter with respect to average filters for signal detection can also be found in [15].

The size of Median Filter window is an odd number, which can be $3,5,7, \cdots,(2 k+1)$. From our experiments, a window size of 5 has been proved to be the most appropriate.

The combination of multi-frame spectrum fusion and adaptive CFAR detection makes the whole detection system extremely robust and reliable. As boat-generated signals have relatively long duration, we have used a multi-frame spectrum fusion, i.e. time-integration over multiple frames which significantly improves the SNR. Although this step introduces a delay in early detection of incoming boats in the order of 15 seconds, this is completely negligible with respect to the typical travelling speeds of monitored boats. The Adaptive Median Filter Constant False Alarm Rate (AMCFAR) algorithm is used to detect boat signature with relatively high detection rate while maintaining a low and constant false alarm rate.

\section{EXPERIMENTAL RESUltS OF TRACKS MATCHING AND ADAPTIVE SignATURE DeTECTION AlgORITHM BASED ON THE MULTI- FRAME FUSION}

The test signals are provided by Soncom PTY LTD from "C-Buoy/Off-Buoy Processor Sea Trials' at Low Islets, Australia $\left(16.3833^{\circ} \mathrm{S}, 145.5667^{\circ} \mathrm{E}\right)$ on 17 June 2002 . The proposed tracks matching, multi-frame spectrum fusion and adaptive signature detection algorithms have been successfully tested on several real ship generated signals in the following, which include the signals called "Naiad1" and "SF6" for reference. 


\subsection{SPECTRUM SIGNATURE DETECTION TEST}

The proposed algorithms have been coded in Matlab, and the signature detection results have been shown in Figs. 4 and 5. Fig. 4 (a) shows us that the "Naiad1" boat signal has a pretty wide frequency band with a quite weak strength, which spreads between about 40 to $1 \mathrm{k} \mathrm{Hz}$, with the main frequency component at about $200 \mathrm{~Hz}$ and the strength of these frequency components between 0.5 to $4 \mathrm{~dB}$. Fig. 4 (b) shows us the binary detections based on the Median CFAR threshold, in which the floating CFAR threshold vector has adapted to its original normalized multiple spectrum fusion vector to avoid any false detections. Fig. 4 (c) shows us the detected spectrum signature of "Naiad1" in the observation period with the normalized multiple frame spectrum vector, in which we can see that the spectrum signature has been reliably detected without any false detections.

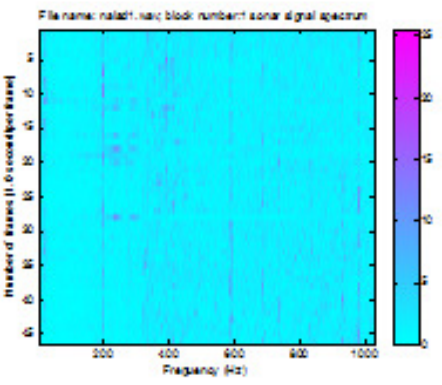

(a) Naiad1 Boat Multi-Frame Signal Spectrum.

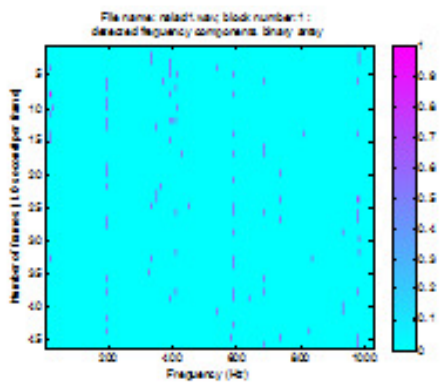

(b) Detected target frequencies by using the proposed Median CFAR algorithm with window size of 5

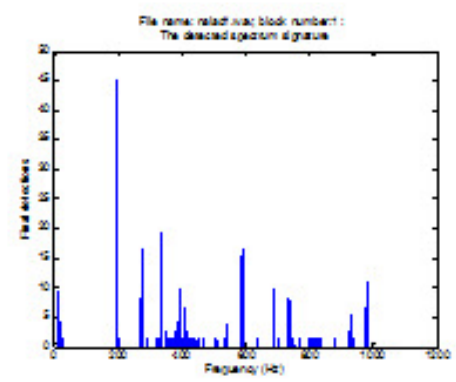

(c) The detected spectrum signature

Figure 4 Experimental results of "Naiad1" with the adaptive median CFAR algorithm with the sliding window size of 5, and proposed multiple frame fusion algorithms (block size: 45 ; initial frame number: 40).

\section{2. "SF1" BOAT SIGNAL TEST}

The proposed Adaptive Median CFAR detection and Multi-Frame spectrum fusion Algorithms have also been tested on "SF1" " boat signal, and the test results are shown in Fig. 5. Fig. 5 (a) shows us that the "SF1" boat signal has also a pretty wide frequency band, which spreads between about 50 to $380 \mathrm{~Hz}$, and 700 to $900 \mathrm{~Hz}$, with the main frequency component at about 200 $\mathrm{Hz} 750 \mathrm{~Hz}$, and the strength of these frequency components between 5 to $25 \mathrm{~dB}$. Fig. 5 (b) shows us the binary detections with the Median CFAR threshold, in which the floating CFAR threshold vector has adapted to its original normalized multiple spectrum. accumulated spectrum fusion vector, in which the SNR has been significantly improved, and it also shows us the normalized multiple spectrum fusion vector (blue) vs its fusion vector to avoid any false detections. Fig. 4 (c) shows us the detected spectrum signature of "SF6" in the observation period (red) with the normalized multiple frame spectrum fusion vector (green), in which we can see that the spectrum signature has been reliably detected without any false detections. The overall procedure is computationally light, thus allowing us cost-effective real-time implementation even on systems with limited computational power and size constraints such as on-board embedded computers. 


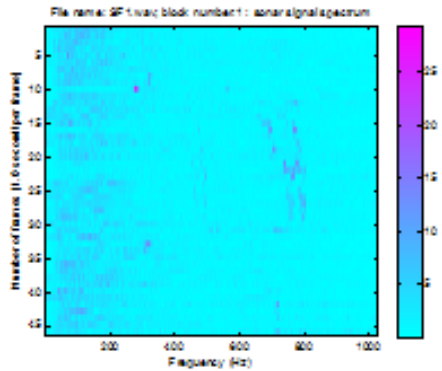

(a) SF1 Boat Multi-Frame Signal Spectrum

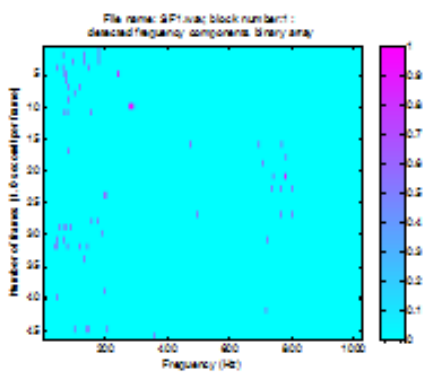

(b) Detected target frequencies by using the proposed Median CFAR algorithm with window size of 5

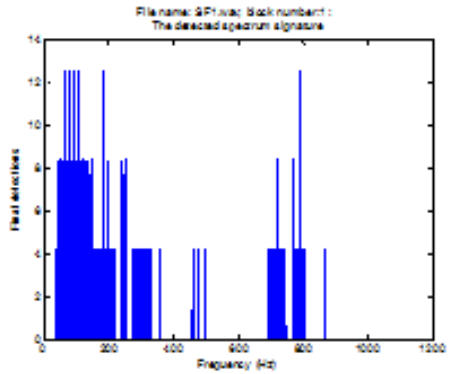

(c) The detected spectrum signature.

Figure 5 Experimental results of "SF1" of the proposed multiple frame spectrum fusion and adaptive median CFAR algorithm with sliding window size of 5. (block size: 45; initial frame number 10)

\subsection{TRACKS MATCHING BASED ON MANHATTAN DISTANCE- "NAIAID1" VERSUS “SF1" SIGNALS}

The proposed tracks matching algorithm has been tested on signal "Naiad1" and "SF1". Each track has 9 blocks, and each block is consisted of 15 frames, and the spectrum signature of each block is calculated based on the single fame detection and fusion in the same block. The spectrum signatures of the two tracks are shown in Table 1 (a), (b) and (c).

Table 1(a). Detected block spectrum signatures (block size: 15 frames; number of blocks: 9).

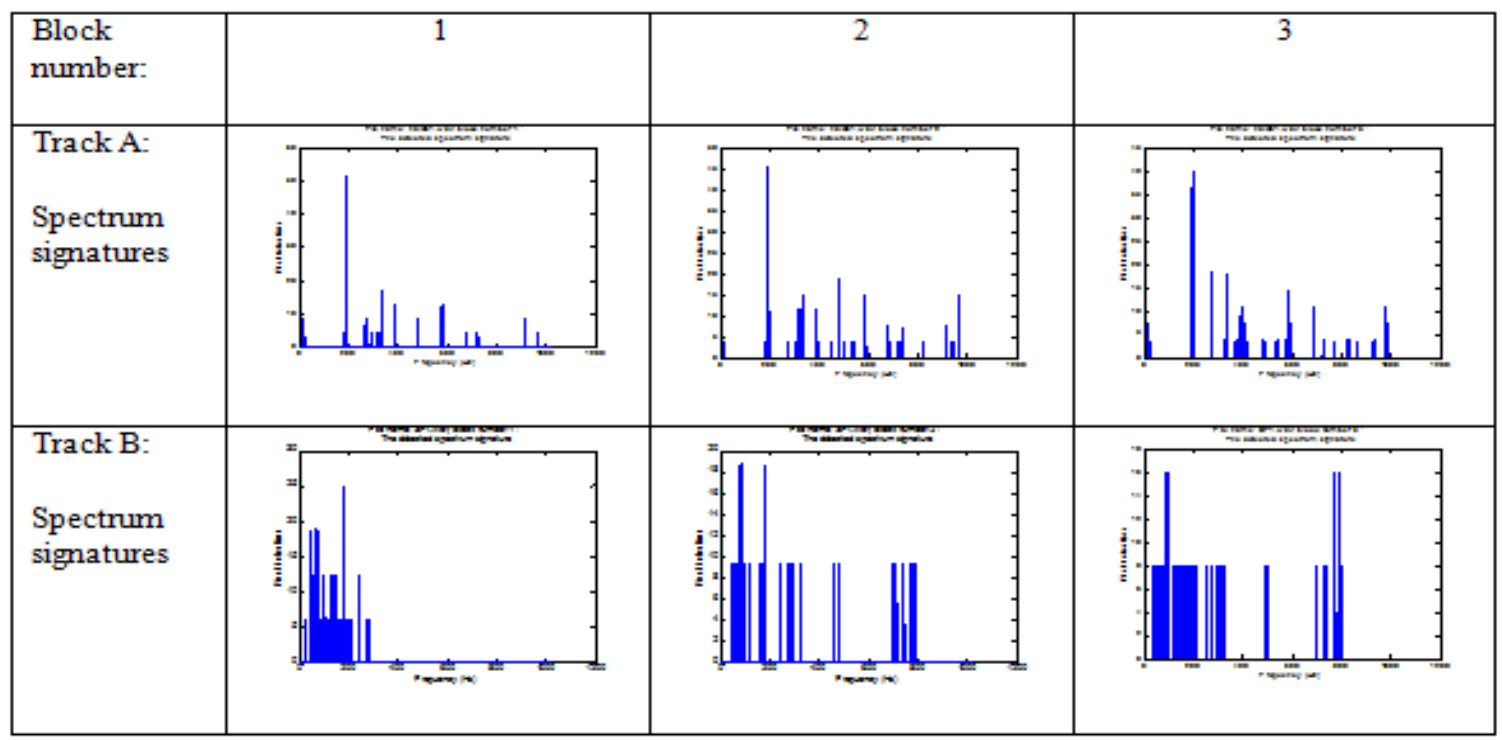


Table 1(b). Detected block spectrum signatures (block size: 15 frames; number of blocks: 9).

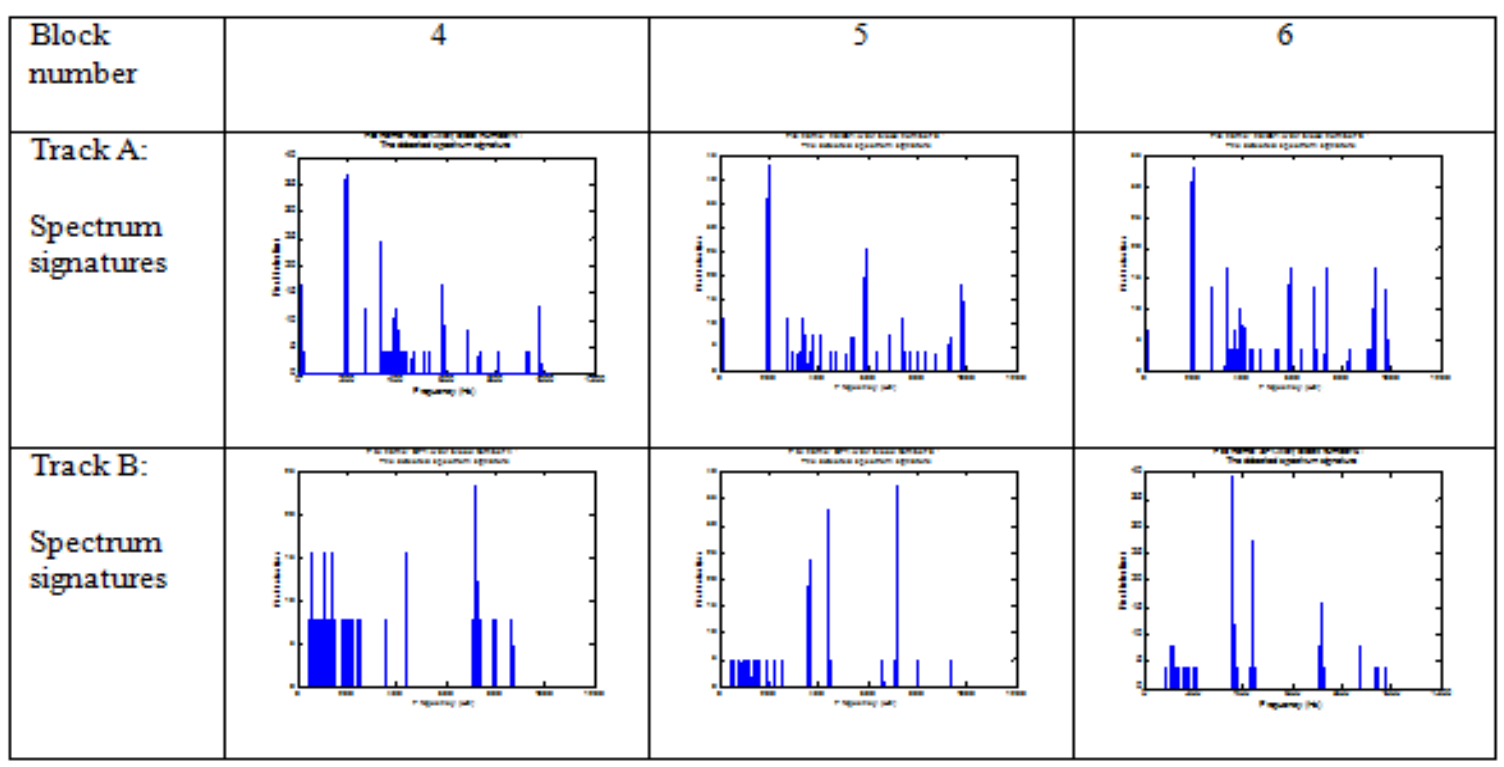

Table 1(c). Detected block spectrum signatures (block size: 15 frames; number of blocks: 9).

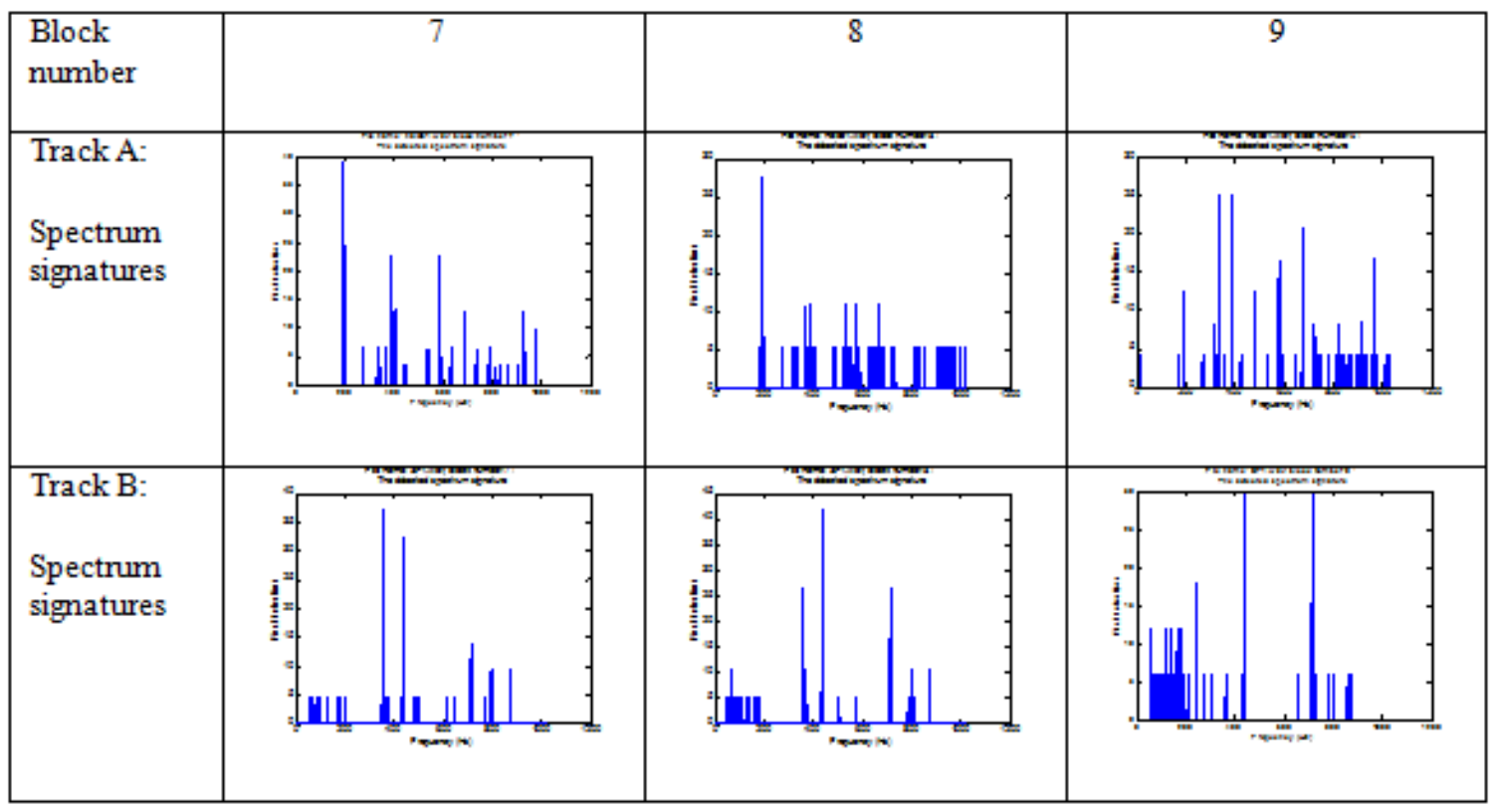

In Table 1, we can see that the spectrum signatures in two tracks of different ships are quite different, and the similarities of the spectrum signatures from the same ship are much more than that from the different ships, but there are also some variations in the same track.

The calculated Manhattan distances between the two tracks in Table 1 are shown in Table 2 and Fig. 6. 
Table 2. The Manhattan distances between Track 1 (Naiad1) and Track 2 (SF1)

\begin{tabular}{|l|l|l|l|l|l|l|l|l|l|}
\hline & Block 1 & Block 2 & Block 3 & Block 4 & Block 5 & Block 6 & Block 7 & Block 8 & Block 9 \\
\hline $\begin{array}{l}\text { Naiad1/ } \\
\text { SF1 }\end{array}$ & 1547.18 & 1685.19 & 1829.72 & 1549.87 & 1367.08 & 1585.52 & 1575.00 & 1964.62 & 1912.40 \\
& & & & & & & & & \\
\hline
\end{tabular}

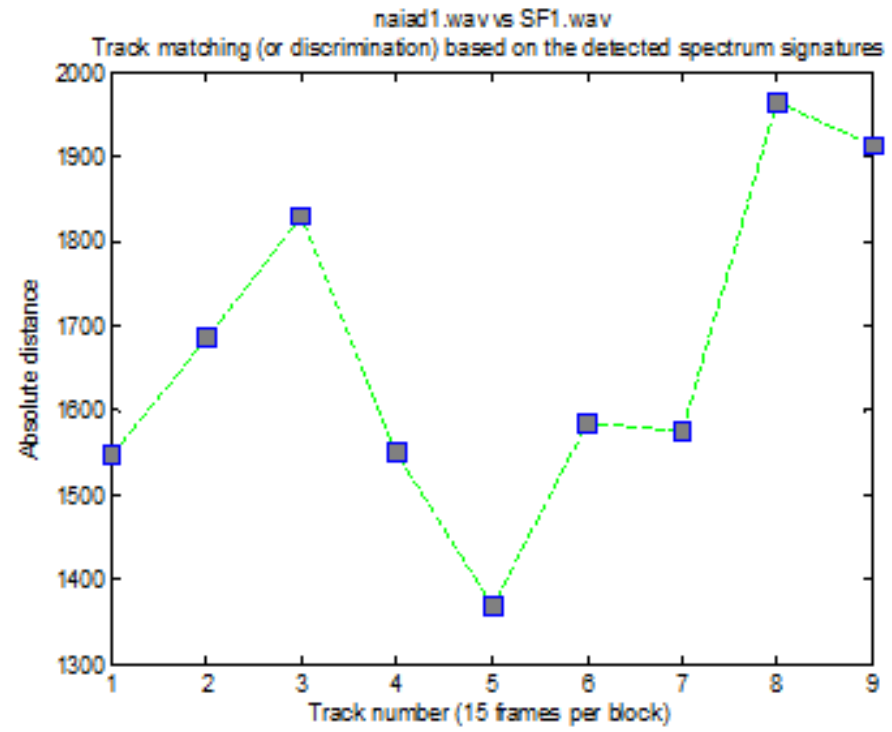

Figure 6. The Manhattan distances between Track 1 (Naiad1) and Track 2 (SF1)

Table 2 and Figure 6 show us that the Manhattan distances between Track 1 (Naiad1) and Track 2 (SF1) are around 1600.

\subsection{TRACKS MATCHING BASED ON EUCLIDEAN DISTANCE- "NAIAID1" VERSUS "SF6" SIGNALS}

The calculated Euclidean distances between the two tracks in Table 1 are shown in Table 3 and Fig. 7.

Table 3. The Euclidean distances between Track 1 (Naiad1) and Track 2 (SF1)

\begin{tabular}{|l|l|l|l|l|l|l|l|l|l|}
\hline & Block 1 & Block 2 & Block 3 & Block 4 & Block 5 & Block 6 & Block 7 & Block 8 & Block 9 \\
\hline $\begin{array}{l}\text { Naiad1/ } \\
\text { SF6 }\end{array}$ & 139.11 & 141.42 & 140.58 & 140.51 & 140.45 & 139.93 & 139.90 & 138.52 & 140.71 \\
\hline
\end{tabular}

Table 3 and Figure 7 show us that the Manhattan distances between Track 1 (Naiad1) and Track 2 (SF1) are around 140. 


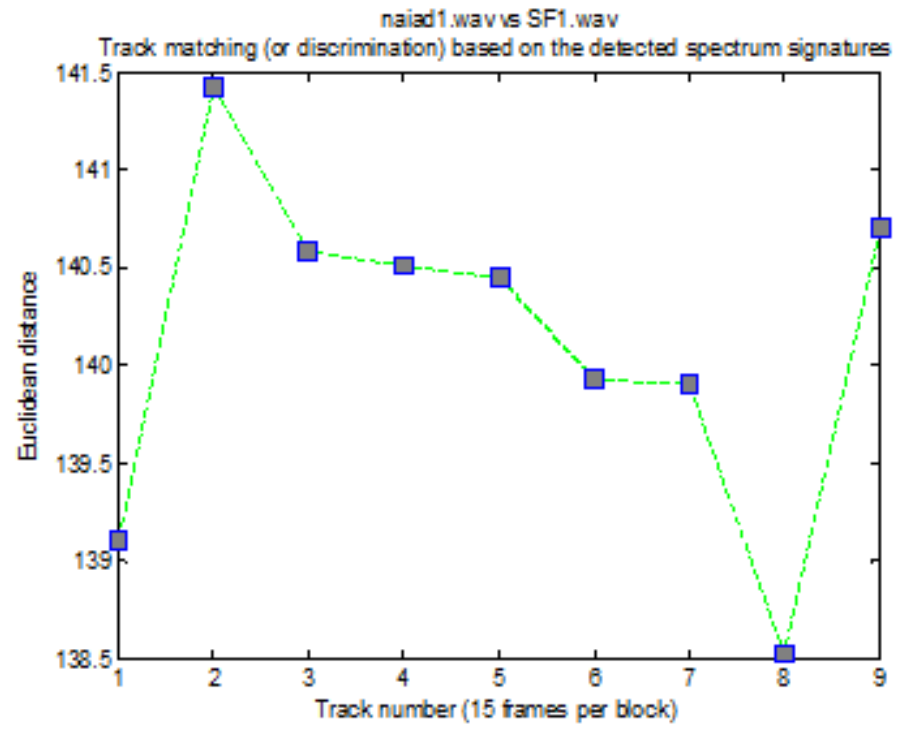

Figure 7. The Euclidean distances between Track 1 (Naiad1) and Track 2 (SF1)

\subsection{TRACKS MATCHING BASED ON MANHATTAN DISTANCE- "NAIAID1" VERSUS "NAIAID1" SIGNALS}

The matching of two tracks that are actually the same target ship is tested by using the data from the same track but shifted by about one block.

The calculated Manhattan distances between the two tracks (Naiad1 and Naiad1 by shifting around 1 block) are shown in Table 4 and Fig. 9.

Table 4. The Manhattan distances between Track 1 (Naiad1) and Track 2 (Naiad1)

\begin{tabular}{|l|l|l|l|l|l|l|l|l|l|}
\hline & Block 1 & Block 2 & Block 3 & Block 4 & Block 5 & Block 6 & Block 7 & Block 8 & Block 9 \\
\hline $\begin{array}{l}\text { Naiad1/ } \\
\text { Naiad1 }\end{array}$ & 251.19 & 395.77 & 239.82 & 392.91 & 282.74 & 312.61 & 303.35 & 769.51 & 547.03 \\
\hline
\end{tabular}

Table 4 and Figure 8 show us that the Manhattan distances between Track 1 (Naiad1) and Track 2 (Naiad1) with 1 block shift) are around 300. 


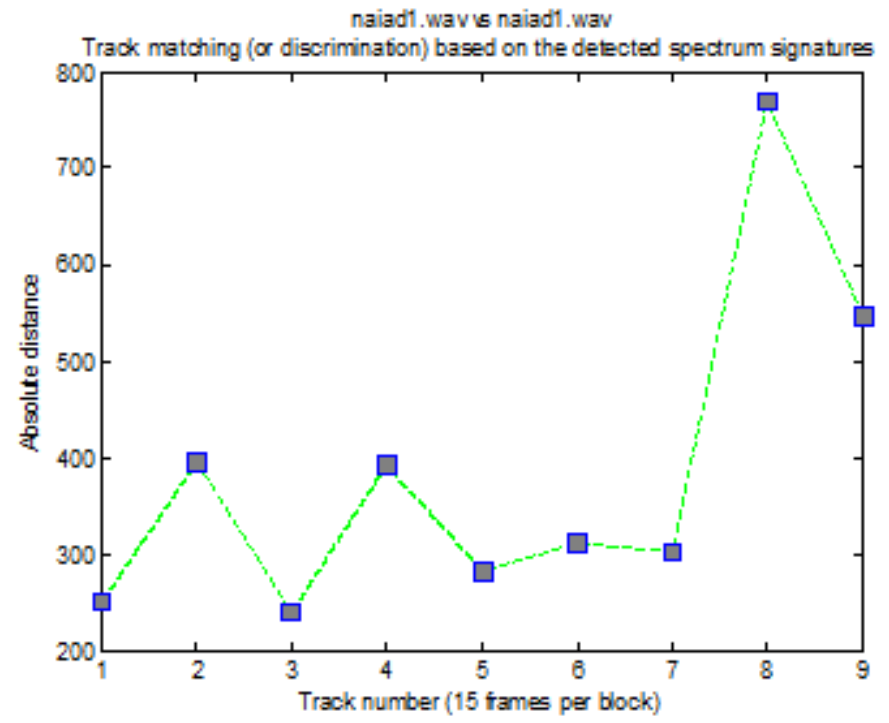

Figure 8. The Manhattan distances between Track 1 (Naiad1) and Track 2 (Naiad1) (with 1 block shift)

\subsection{TRACKS MATCHING BASED ON EUCLIDEAN DISTANCE- "NAIAID1" VERSUS “NAIAID1" SIGNALS}

The calculated Euclidean distances between the two tracks (Naiad1 and Naiad1 by shifting around 1 block) are shown in Table 4 and Fig. 9.

Table 5. The Euclidean distances between Track 1 (Naiad1) and Track 2 (Naiad1)

\begin{tabular}{|l|l|l|l|l|l|l|l|l|l|}
\hline & Block 1 & Block 2 & Block 3 & Block 4 & Block 5 & Block 6 & Block 7 & Block 8 & Block 9 \\
\hline $\begin{array}{l}\text { Naia d1/ } \\
\text { Naia d1 }\end{array}$ & 34.86 & 48.96 & 33.02 & 46.88 & 35.65 & 39.72 & 38.04 & 81.39 & 51.71 \\
\hline
\end{tabular}

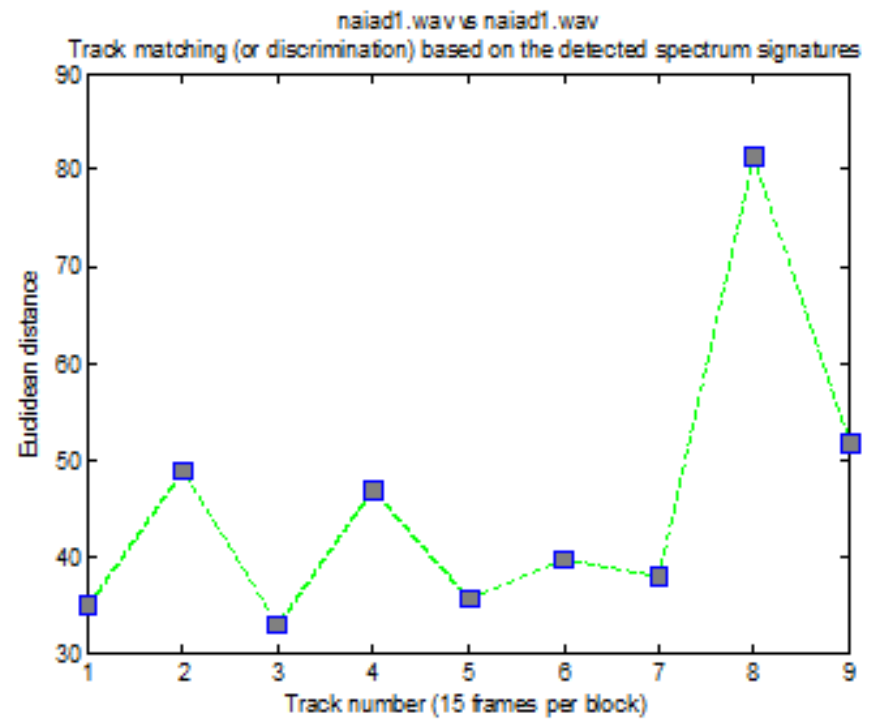

Figure 9. The Euclidean distances between Track 1 (Naiad1) and Track 2 (Naiad1)(with 1 block shift) 
Table 5 and Figure 9 show us that the Euclidean distances between Track 1 (Naiad1) and Track 2 (Naiad1) with 1 block shift) are around 40.

\subsection{TRACKS MATCHING (DISCRIMINATION) BASED ON MANHATTAN AND EUCLIDEAN DISTANCES}

The power of discrimination (difference) or matching (similarity) of proposed algorithms are tested by plotting the Manhattan and Euclidean distances of tracks from different target tracks or from the same target tracks on the same space, drawing a line to separate them.

\section{TRACKS MATCHING (DISCRIMINATION) BASED ON MANHATTAN DISTANCE:}

The Manhattan distances between Track 1 (Naiad1) and Track 2 (Naiad1) (with 1 block shift) is shown in Figure 10 in red lines, and the Manhattan distances between Track 1 (Naiad1) and Track 2 (SF1) are shown in green lines.

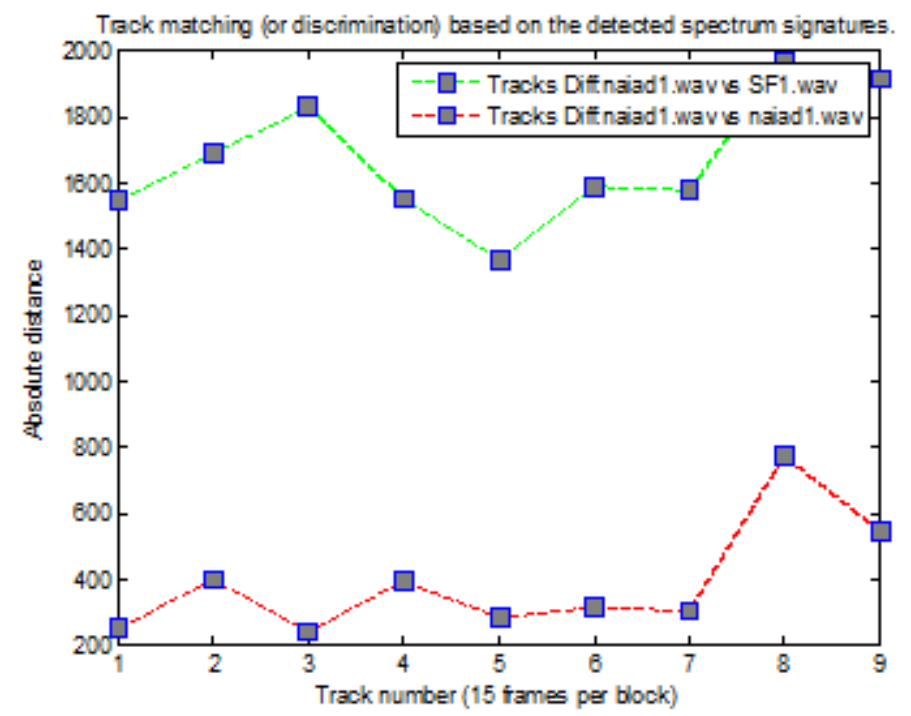

Figure 10. The Manhattan distances between Track 1 (Naiad1) and Track 2 (Naiad1) (with 1 block shift), and distances between Track 1 (Naiad1) and Track 2 (SF1).

Figure 10 shows us that the tracks distances of different target ships "Naiad1" versus "SF1" are around 1600, but the tracks distances of the same target ship "Naiad1" versus "Naiad1" are around 300 . So, by drawing a line around 1000, it is very easy to separate the tracks of the same ship from the tracks from different ships.

\section{TRACKS MATCHING (DISCRIMINATION) BASED ON EUCLIDEAN DISTANCE:}

The Euclidean distances between Track 1 (Naiad1) and Track 2 (Naiad1) (with 1 block shift) is shown in Figure 11 in red lines, and the Euclidean distances between Track 1 (Naiad1) and Track 2 (SF1) are shown in green lines 


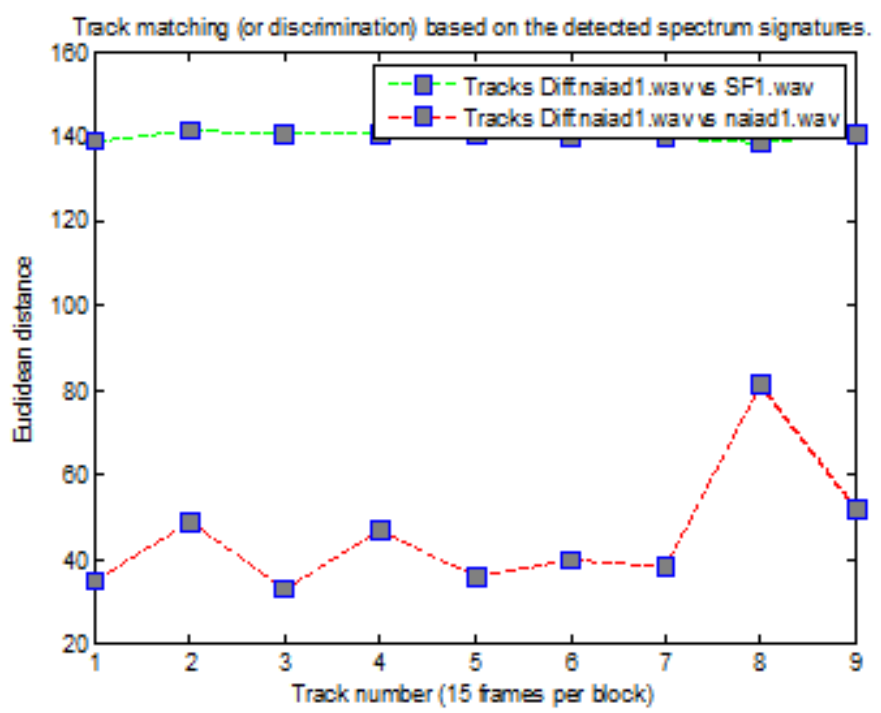

Figure 11. The Euclidean distances between Track 1 (Naiad1) and Track 2 (Naiad1) (with 1 block shift), and distances between Track 1 (Naiad1) and Track 2 (SF1).

Figure 11 shows us that the tracks distances of different target ships "Naiad1" versus "SF1" are around 140, but the tracks distances of the same target ship "Naiad1" versus "Naiad1" are around 40. So, by drawing a line around 90 , it is very easy to separate the tracks of the same ship from the tracks from different ships.

\section{CONCLUSION AND FUTURE WORK}

In this paper, an acoustic spectrum signature tracks matching algorithm based on the Manhattan distance and the Euclidean distance of signature vectors, and a multi-frame fusion algorithm are proposed for reliable real time detection and matching of boat generated acoustic signal spectrum signatures. In which, an adaptive median constant false alarm rate algorithm has been used for effective spectrum signature detection of boat-generated acoustic signals, in which a low constant false alarm rate is kept with relatively high detection rate. The proposed algorithms have been tested on many real acoustic signals recorded from hydrophone at a site on the Australian coastline, two of them are shown in the paper. The statistical analysis and experimental results showed that the proposed algorithms have increased the SNR significantly in the observation period, and have kept a very low false alarm rate and relatively high detection rate for the whole detection system.

The following conclusions can also be drawn:

1) The experiments results have shown that the proposed tracks matching algorithm has the ability to discriminate the tracks from different ships and the ability of matching of the tracks from the same ship; and the spectrum signature detection algorithm has captured the critical features of ship generated acoustic signals.

2) The proposed multi-frame fusion algorithm has increased the SNR significantly, and made the whole detection more robust. 
3) The proposed Adaptive Median CFAR algorithm is used to detect target frequency signature from a multiple frame fusion spectrum vector, keeping our automatic target detection system at low and constant false alarm rate. This algorithm has been proven that it is especially good for detecting LOFAR target frequency components.

4) A magnitude normalization (in the frequency domain) is used to keep our automatic detector more robust to noise and spurious frequencies.

5) With the default sensitivity value, most target frequency components are correctly detected. Further decreasing the sensitivity value makes the false detection rate (alarm rate) lower, but at the same time less target frequency components will be detected.

6) The boat-generated frequency spectrum signature can be detected with high accuracy. In the experiment reported in this paper, the detected boat-generated frequencies of 'Naiad1' and 'SF1' are very close to the "ground truth".

\section{Future work:}

1) Recognition - The detected spectrum signatures can be used for ship recognition and tracking in the future, in which the study of similarity measures between ship spectrum signatures, and the neural network can also be possibly applied for the recognition of detected ships, based on a database of collected spectrum signatures.

2) Tracking-The proposed real time processing and detecting based sonars can be connected into a worldwide undersea network, in which the ships around each sonar can be detected, tracked, and displayed in a control center.

3) Arrays processing - Sonar array processing based on the proposed algorithm as a building block can also be used to increase the SNR of input signal, or detect the direction of incoming ships, [29-35]

\section{ACKNOWLEDGEMENTS}

The research is supported by 2017 Changshu Scientific and Technology Development Plan Project (CQ201701), and the test data was provided by Sonarcom PTY LTD in Australia.

\section{REFERENCES}

[1] Chang Cheini (2000), “An Information Theoretic Approach to Spectral Variability, Similarity, and Discrimination for Hyperspectral Image Analysis", "IEEE Transactions on Information theory, Vol 46, No. 5, 2000, 19271932.

[2] Farifteh, J., Van Der Meer, F., Carranza, E.J.M. , 2006b, Similarity measures for spectral discrimination of saline soils, In Press: International Journal of Remote Sensing.

[3] Van Der Meer, F., 2006, The effectiveness of spectral similarity measures for the analysis of hyperspectral imagery, International Journal of Applied Earth Observation and Geoinformation, 8, pp. 317. 
[4] Du H, C.I. Chang, H. Ren, Cc. Chang, J. O. Jensen, And F. M. D ${ }^{-}$Amico., 2004, New hyperspectral discrimination measure for spectral characterization, Optical Engineering, 43, no 8, pp. 1777" ${ }^{\circ} \mathrm{C} 1786$.

[5] J. X. Qiu, L. Zheng, Y. C. Wang, "Research on ship-radiated noise beat tune", Technical Acoustics, vol. 33, no. 4, pp. 322-325, 2014.

[6] F. S. Zhang, D. Feng, "Fusion and extraction of modulation feature from ship radiated-noise based on wavelet packet and ZFFT", Electronics World, vol. 14, no. 2, pp. 105-106, 2014.

[7] X. W. Luo, S. L. Fang, "Feature extraction from non-stationary amplitude modulated broad-band signal using the Hilbert-Huang transform", Signal Processing, vol. 27, no. 6, pp. 950-955, 2011.

[8] Xueyao Li ; Fuping Zhu; Harbin Eng. and Harbin Univ., Application of the zero-crossing rate, LOFAR spectrum and wavelet to the feature extraction of passive sonar signals, Proceedings of the 3rd World Congress on Intelligent Control and Automation, 2000., vol.4, pp. 2461-2463 (2000).

[9] G. Q. Wu, "Ship radiated-noise recognition (I) the overall framework analysis and extraction of linespectrum", Acta Acoustica, vol. 23, no. 5, pp. 394-400, 1998.

[10] Y. S. Cheng, X. Gao, H. Liu, "A method for ship propeller blade-number recognition based on template matching", Technical Acoustic, vol. 29, no. 2, pp. 228-231, 2010.

[11] Peng H.C., Long F., and Ding C., Feature selection based on mutual information: criteria of maxdependency, max-relevance, and min-redundancy, IEEE Transactions on Pattern Analysis and Machine Intelligence, Vol. 27, No. 8, pp. 1226-1238 (2005).

[12] Roholing, H., "Radar CFAR Thresholding in Clutter and Multiple Target Situations," IEEE Transactions on Aerospace and Electronic System, Vol. AES-19, No. 4, July 1983, pp. 608-621.

[13] S. Wang, J. X. Qiu, S. J. Wang, "Enhancement of ship radiated noise DEMON spectrum SNR based on correlation properties theory of principles of system_dynamics", Ship Science and Technology, vol. 35, no. 8, pp. 24-27, 2013.

[14] Y. S. Cheng, Y. C. Wang, "DEMON analysis of underwater target radiation noise based on modern signal processing", Technical Acoustics, vol. 25, no. 1, pp. 71-74, 2006.

[15] Chan, Y. T., "Underwater Acoustic Data Processing", NATO ASI Series, Kluwer Academic Publishers.

[16] R. O. Nielsen, “Sonar Signal Analysis”. Boston, MA, Artech House, 1991, pp. 123-128.

[17] Merrill I, Skolnik, "Introduction to Radar Systems". McGraw-Hill Book Company, 1980.

[18] Eric Dahai Cheng, Massimo Piccardi and Tony Jan, "Stochastic Boat-Generated Acoustic Target Signal Detection in Time-Frequency Domain", IEEE International Symposium on Signal Processing and Information Technology (ISSPIT'04), Rome, Italy, December, 2004.

[19] Stergios Stergiopoulos, “Advanced Signal Processing Handbook”, CRC Press.

[20] Burdic, W. S., 1984, “Underwater Acoustic System Analysis”, Prentice-Hall, Englewood Clissf, NJ.

[21] Waite A. D., Sonar for practical Engineers, John Wiley, 3rd Ed. (2003). 
[22] H. L.Van Trees, "Detection, Estimation and Modulation Theory". Part I. New York, Wily, 1968, pp. 68-85.

[23] Soares Filho, W.; Manoel de Seixas, J.; Pereira Caloba, L., Principal component analysis for classifying passive sonar signals. International Symposium on Circuits and Systems, Sydney, Australia, vol. 2, pp. 592-595 May (2001).

[24] Soares Filho W., Seixas J. M. and de Moura N. N., Preprocessing passive sonar signals for neural classification. IET Radar, Sonar \& Navigation, vol. 6, pp. 1-14 (2011).

[25] 1. R. J. Urick, Principles of Underwater Sound (3rd Edition), Washington, USA: McGraw-Hill, 1983.

[26] R. O. Nielsen, Sonar Signal Processing, MA: Artech House Inc., 1991.

[27] J. C. D. Martino, J. P. Haton and A. Laporte, "Lofargram line tracking by multistage decision," in Speech, and Signal Processing, USA, 1993. Lee, S.hyun. \& Kim Mi Na, (2008) "This is my paper", ABC Transactions on ECE, Vol. 10, No. 5, pp120-122.

[28] Johnson, D. H.; Dudgeon, D. E. (1993). Array Signal Processing. Prentice Hall.

[29] Van Trees, H. L. (2002). Optimum Array Processing. New York: Wiley.

[30] Krim, H.; Viberg, M. (July 1996). "Two Decades of Array Signal Processing Research" (PDF). IEEE Signal Processing Magazine: 67-94. Retrieved 8 December 2010.

[31] S. Haykin and K.J.R. Liu (Editors), "Handbook on Array Processing and Sensor Networks", Adaptive and Learning Systems for Signal Processing, Communications, and Control Series, 2010.

[32] E. Tuncer and B. Friedlander (Editors), "Classical and Modern Direction-of-Arrival Estimation", Academic Press, 2010.

[33] Prof. J.W.R. Griffiths, Adaptive array processing, IEEPROC, Vol. 130,1983.

[34] N. Petrochilos,G. Galati, E. Piracci, Array processing of SSR signals in the multilateration context, a decade survey.

\section{AUTHORS}

Dahai Cheng (BE 1982, ME 1986, and PhD 1999) was born in 1961, in Xian, China; who is currently a professor at Changshu Institute of Technology. His research interests are radar and sonar signal processing, biological signal and image processing, computer vision, video surveillance and pattern recognition etc. He has about 40 academic papers published in recognized international journals and international conferences. He had been working on several ARC projects in NICTA, UTS, UNSW, and several R\&D projects in Sonacom and MDA etc. He

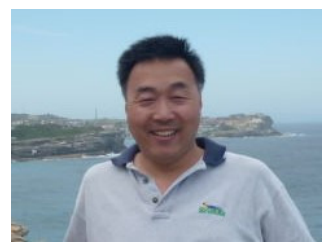
was also a specially invited professor at Xian University of Posts \& Telecommunications.

E-mail: ericdahaicheng@qq.com 
Huigang Xu was born in September 1969; who graduated from the Department of Applied Physics in East China Normal University (Bachelor degree), and obtained his PhD from Nanjing University of Science and Technology in control science and engineering in 2007, and currently he is a professor at Changshu Institute of Technology. He was a young leader of science and technology group in "333 highlevel personnel training project", and he was also selected as an "outstanding young teacher" in Jiangsu province, and a leader of key discipline project in the university. Professor $\mathrm{Xu}$ is a director of electrical and automation specialized committee in China Automation Society; and a director of electronic control system and device

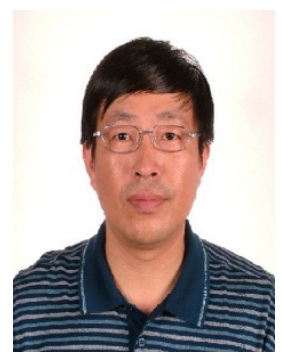
specialized committee, in China Electrotechnical Society; and also a director of Jiangsu automation society. Professor $\mathrm{Xu}$ is a master degree postgraduate supervisor of Suzhou University and China University of Mining and Technology, and is mainly engaged in the research work in signal detection and control, industrial automation, and has completed of 2 National Natural Science projects, and 2 provincial prospective research projects, and received a Jiangsu Provincial Science and Technology Progress Award, and a National Machinery Industry Science and Technology Progress Award.

E-mail: xuhuigang@cslg.edu.cn

Ruiliang Gong was born in November 1965, and currently the CEO of Changshu Ruite Electric Co. Ltd; finished his University Diploma from Changshu Institute of Technology in 1987. He joined Changshu Research Institute of Electronics in 1987, where he was engaged in project research and development, and jointly developed the "coal mine environmental monitoring system" with China University of Mining and Technology, which has passed the user assessment and identification. In 1989, $\mathrm{He}$ served as director of Changshu Research Institute of Electronics, and the director of the electronic instrument test workshop, and in charge of a R\&D of "XXXH2 security monitoring alarm system", and won the "Jiangsu Province Science Technology Progress Award in 1992. Mr Ruiliang Gong was also in charge of the projects of "DH-UPS Navigation UPS" and "GLX marine auxiliary boiler control device" etc.

E-mail: gongrl@cs-ruite.com

Huan Huang was born in August, 1981; who is currently the chief engineer in Changshu Ruite Electric Co. Ltd. Mr Huang graduated from Department of Electrical Engineering, Tongji University (Bachelor) in 2004, and he obtained his master degree in automatic control from Tongji University in 2007. Mr Huang joined Changshu Ruite Electric Co. Ltd in 2008, first as the electronic engineer, then automation department manager, and deputy chief engineer. He has presided over the development of a set of electric field control devices, obtained five invention patents, and won the first prize of Suzhou civil military integration project, and currently involved in drafting a national standard for ships.

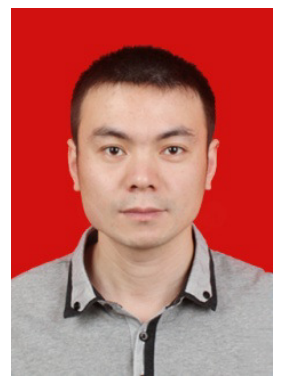

E-mail: 75189915@qq.com 